\title{
The Art of Data Evaluation
}

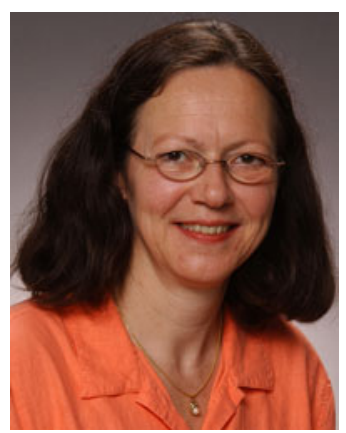

Computational methods are becoming increasingly important in materials science and engineering. It may be human nature that one tends to trust computed data because they are the results of computations, i.e., mathematics, an exact science. However, one must not forget that the results of computations are the results of a specific method or model and, within this specific method, the results reflect the user's choice of input parameters. Therefore, it is quite possible that results from different sources of calculations of a specific property on a specific material under specific conditions are different. This does not necessarily mean that one of the results is wrong, but the knowledge of the computational details is essential for comparing data from different calculations.

No matter whether data are experimental or derived from computations, a critical evaluation needs to be performed to gain understanding of a system. It is imperative that the original data are used in a critical evaluation since each new generation of the data can introduce errors and these errors may propagate. The Ag-Cu-Pb system (Ternary Alloys, Vol. 2, VCH, Weinheim, Germany, 1988) is a good illustration. The evaluation shows two liquidus projections, one based on experimental data and one from a CALPHAD calculation. The difference between the two is the extent of the miscibility gap in the liquid phase. The evaluated liquidus projection is dominated by the miscibility gap originating from the binary $\mathrm{Cu}-\mathrm{Pb}$ system while in the calculated liquidus projection this miscibility gap barely extends into the ternary system. The evaluated liquidus projection was taken from an earlier evaluation (ICRA Monograph VI, 1979) which was taken from an even earlier evaluation (Kurzgefasstes Handuch aller Legierungen, Otto Spammer Verlag, Leipzig, Germany, 1937). The puzzling fact is that the 1937 evaluation and the calculation are based on the same experimental data (Friedrich and Leroux, Metallurgie, 4 (1907) 293). The explanation for the difference can be found in the 1937 evaluation where the experimental data were reinterpreted to match the then accepted phase diagram of the $\mathrm{Cu}-\mathrm{Pb}$ subsystem where the miscibility gap was greatly overestimated. The authors of the calculation of the ternary system selected the original experimental data for their assessment, thus avoiding propagation of the error.
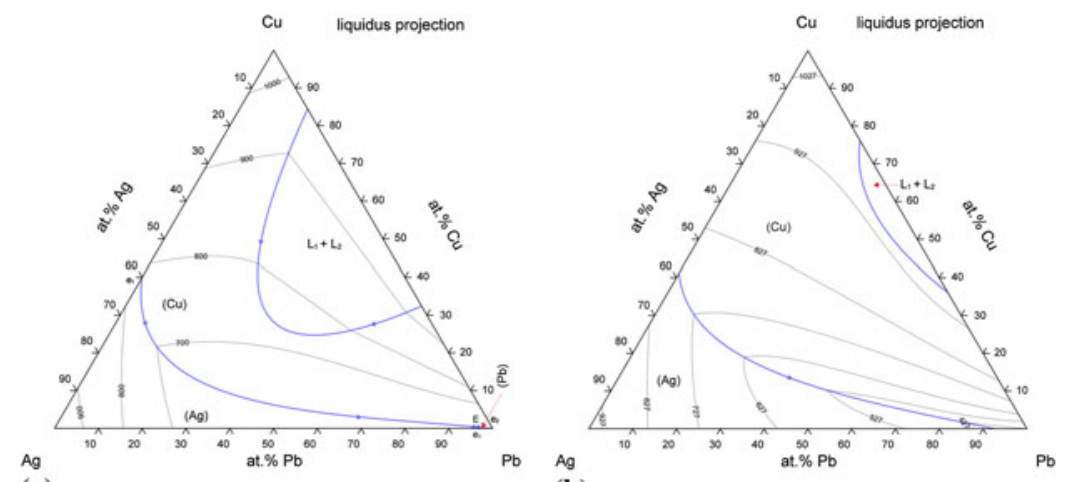

(a)

(b)

Evaluated (a) and calculated (b) liquidus projection of the $\mathrm{Ag}-\mathrm{Cu}-\mathrm{Pb}$ system (Downloaded from http://www.springermaterials. com/docs/VSP/summary/lpf-c/00000167.html?queryterms="ag-cu-pb")

In addition to using the original work for the data evaluation, it is also important to use the originally measured data since they are free of accumulated errors, errors in data conversion or errors in interpretation. For example, one standard procedure for measuring the enthalpy of mixing of the liquid phase is to start with one pure element, add the second pure element to form an alloy and then continue adding the second element. The temperature change in the calorimeter is recorded for each addition and then used to calculate the enthalpy changes caused by the additions of the second element. Integral enthalpies reported from such measurements may carry errors that are accumulated from measurement to measurement, and also may be affected by the choice of reference, which is used to correct for temperature differences between the alloy and the element being added (Zakulski et al., J. Phase Equilib. 14 (1993) 184). Unfortunately, the originally measured data are not always reported and the information given may be inadequate to reconstruct the originally measured data. However, this needs to be taken into account in a critical evaluation when the reliability of data is judged.

The ability to critically screen the available data using the original publications is the foundation for a good evaluation. No matter whether data have been obtained from experiments or computation, it is imperative that details on how these data were obtained are reported.

\author{
Ursula R. Kattner \\ Associate Editor \\ Journal of Phase Equilibria and Diffusion
}

